\section{Selecting the best regression equation by maximizing double cross-validation correlation}

\author{
JOHN D. MORRIS \\ Department of School Services, Box 8143 \\ Georgia Southern College, Statesboro, Georgia 30458
}

Tatsuoka (1973, p. 279) has suggested that the ultimate criterion for selecting a subset of predictor variables from those available is cross-validation, or applying the regression weights obtained in one sample to an independent sample, and then to calculate the correlation of the predicted criterion with the actual criterion (weight validity). For this reason, he suggests that an equation obtained by one of the typical variable selection methods (forward, backward, or stepwise selection) should be cross-validated to obtain an estimate of the degree of relationship to be expected in subsequent applications. Kerlinger and Pedhazur (1973, p. 284) have described double crossvalidation as a more rigorous approach. In this method, the separate equations obtained in two samples are each applied to the alternate sample. In this case, a weight validity can be calculated separately for each sample, or can be calculated over both samples.

In addition to these admonitions, in terms of the generality of the best variable subset selected by typical procedures, certain other problems may arise. Specifically, the variable subset chosen by a selection technique may not be the same as would be selected by another technique, and, within a technique, the subset selected depends on the probability level chosen for the necessary tests of inclusion and/or exclusion. Moreover, these inferential tests assume normally distributed variables. A final concern is that, whether variable selection procedures have been employed or not, the typically used estimate of the "shrunken R" is not an estimate of the multiple correlation, or of the weight validity to be expected on application to subsequent samples, but an estimate of the multiple correlation that would have existed if the population regression weights had been used rather than the sample-generated least-squares estimates (Burket, 1964). Since interest is usually and justifiably focused on the level of relationship to be expected in subsequent samples for which the criterion measure is unknown, this index may be of limited utility. The purpose of this paper is to describe a methodology and accompanying computer program which will select a multiple regression equation optimizing the double cross-validation weight validity and also yielding weightvalidity estimates to be expected in future applications, and their significances.

Computational procedure. The input sample with $p$ predictors is randomly split in half and least-squares-estimated regression weights are calculated for all $2^{p}-1$ possible regression equations for each sample. The weight validities for each equation over both subsamples are then calculated. The equation manifesting the highest weight validity is noted. This procedure is repeated either an input number of times or until a regression equation shows itself superior. The superiority of the

This research was funded in part by a Georgia Southern College Faculty Research Grant. regression equation is judged in terms of the binomial probability that the number of times the superior equation showed the highest weight validity is equivalent to the number of times the next best (equivalently, all inferior equations) equation was highest. If the probability thus generated is smaller than a user input probability, then the null hypothesis is rejected, the superior equation is chosen as best, and iteration stops. Unlike typical variahle selection procedures, normality assumptions are unnecessary. The possibility that a cluster of equally superior equations arise is also accommodated. Such a cluster would be defined by no significant differences, again through the binomial expansion, between the equations within the cluster and each equation being significantly superior to those equations not in the cluster. This incorporates the fact that there may be more than one "best" equation in terms of weight validity. The selection decision from such a set of equations would normally be made on the economic and/or subjective merits of the measurement process involved with each of the variables. Lacking subjective reasons for preferring certain variables over others, the equation within the superior set with the smallest number of variables would probably be selected.

Input. The user must enter a variable format for reading the subjects' profiles, the number of subjects, the number of independent variables, the subjects' input unit number, the probability desired to stop iterations (alternatively the number of iterations desired can be entered and no stopping tests will be made), and an eight-digit seed number for the random-number generator.

Output. The program output includes the means, standard deviations, and the intercorrelation matrix for the predictors and criterion calculated on the total sample. For all $2^{\mathbf{p}}-1$ possible regression equations, the mean (Fisher $z$ transformed) weight validity, associated test of significance, and number of iterations for which the weight validity was highest is output. If a probability for stopping iterations was entered, the multiple regression equation and multiple correlation calculated on the total sample for the best equation(s) are output.

Computer and language. This FORTRAN IV program is designed to handle up to 10 independent variables; however, this dimension can be increased with most large systems. The program is in use on a CDC CYBER 74 in both batch and interactive modes, but, with the deletion of one statement, is convertible to IBM 360-70 hardware.

Availability. The program with complete documentation, sample input, and output is available at no cost on request from the author at: Department of School Services, Box 8143, Georgia Southern College, Statesboro, Georgia 30458.

\section{REFERENCES}

BURKer, G. A study of reduced rank models for multiple prediction. Psychometric Monographs, 1964, 12.

Kerlinger, F. N., \& Pedhazur, E. J. Multiple regression in behavioral research. New York: Holt, Rinehart, \& Winston, 1973.

TatsuokA, M. M. Multivariate analysis in educational research. In F. N. Kerlinger (Ed.), Review of research in education, I. Itasca, Illinois: Peacock, 1973. 